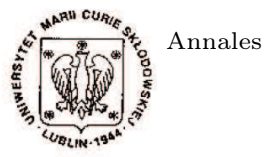

UMCS Informatica AI XIV, 3 (2014) 43-51 DOI: $10.2478 /$ umcsinfo-2014-0017

\section{Annales UMCS}

Informatica

Lublin-Polonia

Sectio AI

http://www.annales.umcs.lublin.pl/

\title{
Electroencephalographic detection of synesthesia
}

\author{
Anna Gajos ${ }^{1 *}$, Grzegorz M. Wójcik ${ }^{1 \dagger}$ \\ ${ }^{1}$ Institute of Computer Science, Maria Curie-Sklodowska University, \\ Akademicka 9, 20-033 Lublin, Poland
}

\begin{abstract}
In this paper the research on a person declaring synesthetic abilities will be presented. According to the current state of knowledge synesthesia activates additional cortical fields in the brain which can be found in the EEG. The research was conducted using an EGI-EEG system (Electrical Geodesic Inc., Eugene, Oregon, USA) with the GeoSource software. GeoSource is a tool that implements the algorithms LAURA, LORETA and sLORETA. Using these algorithms for EEG analysis we can determine where in the brain the source of activity is. The authors will try to answer the question whether the use of these tools can prove the occurrence of synesthesia.
\end{abstract}

\section{Introduction}

The human brain is a complex network of connections in which communication takes place through the conduction of electrical and chemical impulses along dendrites and axons. As a result, on the surface of the brain the activity that we are able to measure using electroencephalograph is formed. This device measures the potential difference between points under two electrodes placed on the scalp, then we take into account measuring of voltage. The resulting record is called electroencephalogram. This is a completely non-invasive way of monitoring brain activity in order to determine what brain is doing at the moment. Then electroencephalogram is defined as the potential difference between two points in time function [1]. EEG signal is generated by the synchronous activity of the inhibitory and stimulating postsynaptic potentials, with the participation of large groups of cortical pyramidal cells [2]. By analyzing the EEG signal the person's current mental state can be determined, one can also distinguish correct from incorrect recording (which is often useful in the diagnosis), as well as

*agajos@hektor.umcs.lublin.pl

†gmwojcik@gmail.com 
determine which parts of the brain communicate with each other at certain mental activities. This is a vast field of research for neuroinformatics. Neuroinformatics is a scientific area where neuroscience combines with computers and often numerical solutions are most appropriate tools for analysis of psychological phenomena $[\mathbf{3}, \mathbf{4}, \mathbf{5}$, 6]. Looking into brain electrical activity may be meaningful for people suffering from a range of disabilities $[\mathbf{7}, \mathbf{8}]$.

In this article, in particular, we deal with the phenomenon of synesthesia. Synesthesia is an extremely rare phenomenon where mixing of sensations is induced by stimuli going through different senses. People diagnosed with synesthesia can for example see sounds and taste colours. Initially, these impressions were attributed to the imagination [9], but after analyzing the distribution of cortical centers in the brain it is supposed to have an neuroanatomical analysis.

Cortical fields responsible for completely different functions may be in its close distance in the brain $[\mathbf{1 0}, \mathbf{1 1}, \mathbf{1 2}, \mathbf{1 3}]$. As a rule, they do not interact with each other, but in about $2 \%-4 \%$ of human population it can lead to communication between them. Then the brain performs certain tasks involving its centers, which should not take part in it [14].

While estimating type of synesthesia, it is customary to put the stimulus of sensations before the induced one. As an example, synesthesia at which we hear a sound and associate it with colour is called phoneme-colour synesthesia and sense of taste due to visual sensations gives grapheme-taste synesthesia.

In this paper the grapheme-colour synesthesia type has been deeply probed. Specifically, it is a crossmodal binding of vision and visual representation of ordinal words (weekdays), which is probably the most common type of synesthesia. Therefore, when analyzing the results, our attention will be attached to the 19 Brodmann area, which is credited with the location of the V4 area. It is believed that this is where the analysis of shapes and colours is conducted [15].

\subsection{Review and research basis}

The fusion of sensations called synesthesia was first scientifically documented in 1812 [16].

The conditions in which synesthetic abilities develop have notbeen fully studied yet. Synesthesia often discloses as a result of brain damage. It is also thought that the presence of synesthesia is genetic [14].

There is no right way to detect synesthesia. There are psychological methods of checking elements that may indicate synesthesia and distinguish it from other phenomena such as visual imaginery. These are the automaticity (synesthetic impression occurs independently of the will), consistency (specific stimulus always evokes the same synesthetic reactions over time), projection (synesthete does not imagine synesthetic experience) and reliability (impression is not associated with memory, like the smell of 
childhood). It is also said that the synesthete approaches the factors causing synesthesia emotionally. Most of psychological methods examine one of these items, so they can not be considered fully reliable [17].

The most common test for detection of synesthesia is a Test Battery and it can be done everywhere in front of a screen. The principle of the test is based on checking coinsistency. If we deal with the type of grapheme-colour synesthesia, the tested person has available colour palette from which he/she chooses the colour corresponding to the grapheme. This must be repeated three times for each grapheme at different timeintervals [18]. But it has a lot of drawbacks and limitations. First of all, the subject usually performs the test independently without any supervision. Besides, synesthete may not associate the grapheme with one particular colour, but see something such as a gradient or irregular texture.

Another type of test is querying of experience for the individual stimulants and repeat the test after a long time. In the case of people with synesthesia, the answers will be the same. There is also a method that checks for automaticity. Subject sees before him a set of letters or numbers in the same colours (for example, 2 and 5), and indicates distinctive scheme among them (for example, the twos are arranged in a triangle). Synesthete will find this model more quickly, because different letters and numbers will be in different colours [17].

Most of the methods are based on a similar or modified rule.

There are also many articles that document the detection of synesthesia by EEG, specifically indicating the activity of certain cortical areas, which theoretically should not take part in the stimulation of grapheme and sound $[\mathbf{1 9}, \mathbf{2 0}, \mathbf{2 1}, \mathbf{2 2}]$. EEG may be a reliable way of detecting synesthesia. Psychological tests can always be questioned, while recorded brain waves can not. We hope to confront this thesis based on the available high-quality electroencephalographic tools and software that implements sLORETA algorithm.

\subsection{Objective}

The study on the type of person declaring grapheme-colour synesthesia has been carried out. In the context of this paper the authors will try to answer the question whether with the 256-channel EGI Geodesic machine with the GeoSource software (sLORETA) it is possible to identify the point at which we deal with the synesthetic impression and in which parts of the brain its source is.

\section{Area of studies}

The study was carried out on high-end hardware of EGI Geodesic Inc.

EGI Geodesics System includes (besides an amplifier with signals from all channels) NetStation 4.5.4 (a software package from EGI for register, review and analysis of EEG signal), E-Prime for NetStation (a software for designing experiments), two machines: one with NetStation and one for running experiments, a computer with eye-tracking 
system SmartEye, sensor nets (EGI's HydroCel Geodesic Sensor Nets with 256 channels) and Geodesic Photogrammetry System - GPS (for improving location of sensors) [23].

In addition, the laboratory is equipped with the Geosource 2.0 software for mapping to a computer model the measured sources of the brain activity to a computer model. It implements 3 algorithms: LORETA (LOw REsolution electromagnetic Tomography), sLORETA (Standardized LOw REsolution electromagnetic Tomography) and LAURA (Local AUtoRegressive Average). All three algorithms are based on the assumption that there are always activated neighboring neurons. As a result of the adoption of the above assumption there are obtained blurry images of the electric charge distribution within the skull [24]. During the EEG analysis for this experiment the sLORETA algorithm was chosen. sLORETA is a method that computes images of electric neuronal activity from EEG and MEG. With the right conditions sLORETA localises a source activity quite accurately. However, it has very low spatial resolution, and spatial resolution decreases with depth [25].

According to Pascual-Marqui: "There are $N_{E}$ instantaneous extracranial measurements. There are $N_{V}$ voxels in the brain. [...] At each voxel there is a point source, which may be a vector with three unknown components (i.e., the three dipole moments), or a scalar (unknown dipole amplitude, known orientation). The cases considered here correspond to $N_{V}>>N_{E} "[\mathbf{2 5}]$.

There are three cases that sLORETA estimates the localization of source activity:

- EEG with unknown current density vector

- EEG with known current density vector orientation and unknown amplitude (cortical surface is completely known, voxels are distributed along the cortical surface, the dipoles at each voxel have known orientation)

- MEG

The main equation of algorithm is:

$\Phi=K J+c 1$,

where $\Phi$ is a vector with electric potentials measured at $N_{E}$ electrodes, $J$ is a current density, where $J$ is a $N_{V}$ - element set of voxels (each voxel contains three unknown dipole moments), $K$ is a matrix $N_{E} \times N_{V}$. Each element of the matrix indicates the electric potential of each voxel, $c 1$ is a constant.

To improve localization of source activity, the algorithm employs the current density estimate given by the minimum norm solution. Then it uses standardization typical of SLORETA.

\section{Methods}

\subsection{Data acquisition}

EEG data were recorded using a 256-channel Geodesic Sensor Net. Data were sampled at $1000 \mathrm{~Hz}$ and recorded using NetStation 4.5.4 (EGI Software: EGI, Eugene, 
OR). Impedances were kept below $100 \mathrm{k} \Omega$ at the beginning of the session. All channels were referenced to $\mathrm{Cz}$ during recording and later rereferenced to an average reference.

A 26-year-old right-handed man (later called S.) has been tested. S. declared synesthetic abilities in the field of viewing days of the week in colours. S. has passed Test Battery.

Before the experiment S. was queried about colours, which he sees on individual days of the week. He used the colour palette. This test was repeated after one year, while S. gave exactly the same answers.

On the basis of that interview an experiment was designed using the ERP software EPrime 2.0. The experiment consisted of watching stimulus in the form of colour and chromatic names of days of the week. The stimulus was composed of a day of the week and the task of the subject was looking at the grapheme. The names of the days of the week were presented as white letters on a black background (A-type grapheme) or in colours. The colours of some stimuli corresponded to those days, which synesthete gave during the initial interview (B-type grapheme). The rest of the stimuli were in random colours, different from those specified by synesthete (C-type grapheme). Additionally, in some stimuli colours given by synesthete were assigned to other days of the week (D-type grapheme). The were 10 samples of each type.

\subsection{Data analysis}

An FIR $0.1-30 \mathrm{~Hz}$ bandpass filter was applied. Data were stimulus-locked to the onset of the test stimulus. Segments were based on $100 \mathrm{~ms}$ pre-stimulus to $700 \mathrm{~ms}$ post-stimulus. For each segment the channel was considered bad if the fast average amplitude exceeded $200 \mu \mathrm{V}$ or eye blink was detected (more than $140 \mu \mathrm{V}$ ) or eye movement occured (more than $55 \mu \mathrm{V}$ ). The rest of ocular artifacts were marked using the SmartEye. Bad channels were replaced through interpolation of neighbouring electrodes [26].

The data were further examined whether the algorithm did not omit any bad segment or trial. Finally, segments were excluded if they contained more than 10 bad channels or eye artefacts.

EEG source imaging was performed using the standardized low resolution brain electromagnetic tomography (sLORETA) method [25], as implemented in the GeoSource 2.0. This method is based on a Sun-Stok 4-Shell Sphere head model with a Tikhonov regularization of $1 \times 10^{-2}$.

\section{Results}

After overlaying evoked potentials of 4 types of stimuli (Fig. 1), one can see that over $700 \mathrm{~ms}$ in the case of D-type graphemes the first reaction to the stimulus was much weaker, but later after the diagnosis of pattern brain reacted strongly. In the case of B-type graphemes the stimuli was quickly recognised and did not raise the reaction outcome, as in other cases. The C-type graphemes waveform look quite similar to 


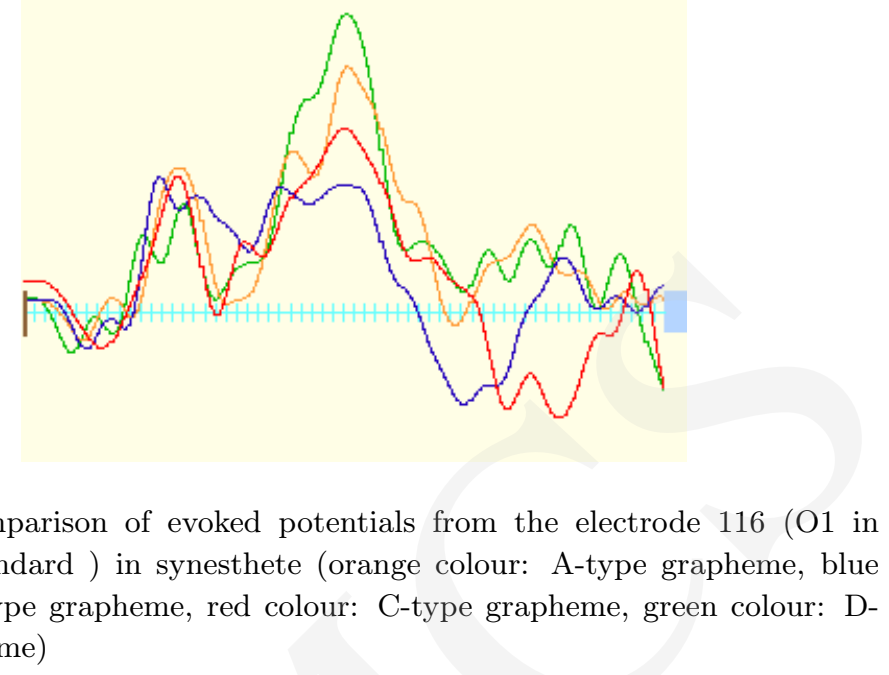

Fig. 1. Comparison of evoked potentials from the electrode 116 (O1 in $10-20$ standard ) in synesthete (orange colour: A-type grapheme, blue colour: B-type grapheme, red colour: C-type grapheme, green colour: Dtype grapheme)

the original signal of B-type graphemes, although it can be seen that the reaction was slower. A-type graphemes signal is similar to the original signal of D-type graphemes, although the reaction was lower after recognition.

On the basis of the signal waveforms visualization was generated - showing the voltage distribution on the surface of the head. Fig. 2 presents TopoMap and Fig. 3 FlatMap generated by the NetStation and GeoSource software. They represent approximately brain response to the stimuli of $100 \mathrm{~ms}$ when looking at a) the chromatic names of days (A-type grapheme), b) the name corresponding to the specified colour (B-type grapheme), c) the names in different colours (C-type grapheme), d) the names of the colours assigned to other days of the week (D-type grapheme).

In Fig. 2a) and 2b) we can see the activity of the 19 Brodmann area, which is located in the area responsible for processing colours and shapes. In Fig. 2c) at the same time, the activity can be seen in the 21 Brodmann area, which is mostly responsible for the audio processing. In Fig. 2d) the reaction is much weaker.

\section{Discussion}

GeoSource helped identify the source of activity, and pointed to the fields that were activated in specific cases. On the basis of GeoSource it can be concluded that synesthete with grapheme-colour reacts differently to the names of days of the week. It depends on how these colours correspond to his personal vision of those days of the week. The 21 Brodmann area is often responsible for audio processing, so it would be assumed that the brain tries to cope with the unusual situation which days of the week have different colours than generally accepted by synesthete. 
Pobrane z czasopisma Annales AI- Informatica http://ai.annales.umcs.pl

Data: 26/04/2023 11:10:27

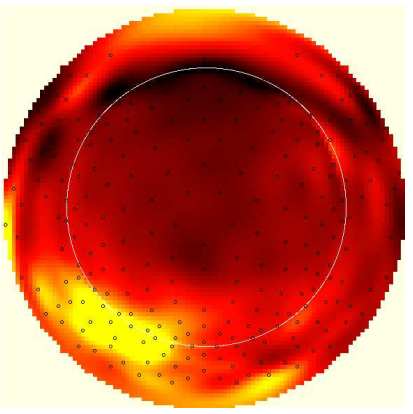

(a) A-type grapheme

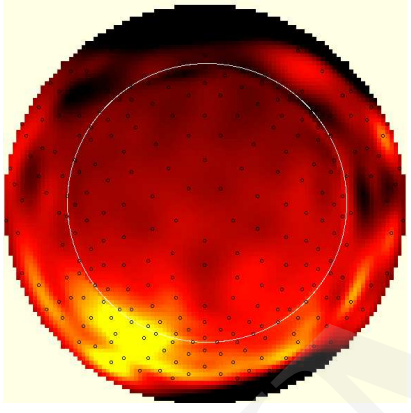

(c) C-type grapheme

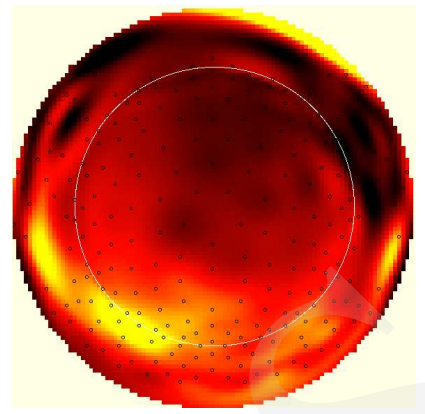

(b) B-type grapheme

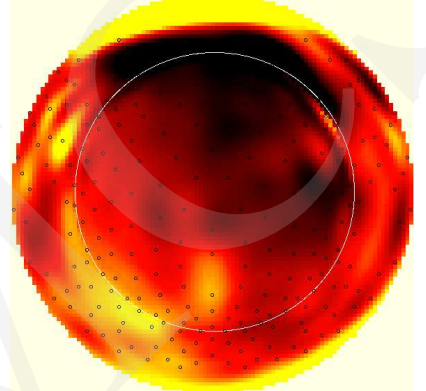

(d) D-type grapheme

Fig. 2. TopoMap in $100 \mathrm{~ms}$ after different stimuli

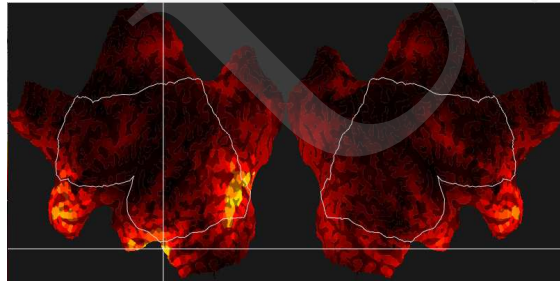

(a) A-type grapheme

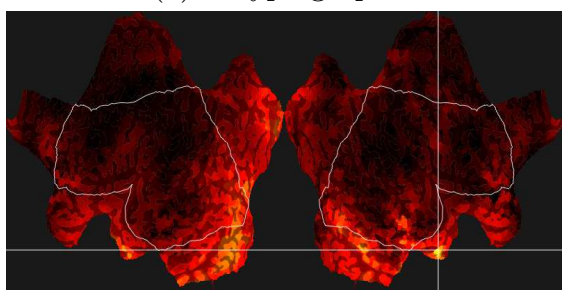

(c) C-type grapheme

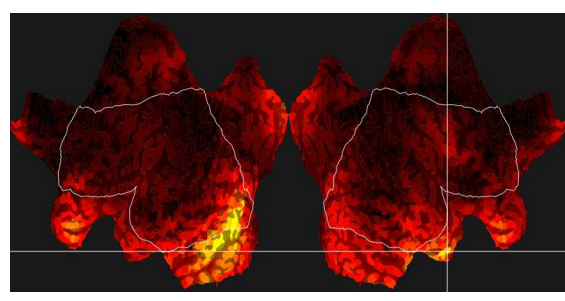

(b) B-type grapheme

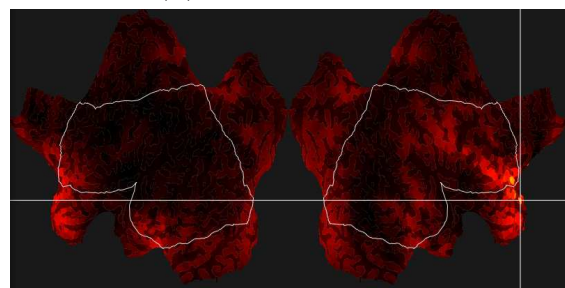

(d) D-type grapheme

Fig. 3. Source activity on Flat Map for different stimuli in synesthete 


\section{Conclusions}

Given that we have 256-channel electroencephalograph and geolocation station, the image of GeoSource can be considered to be relatively accurate. We can use it to identify the main sources of brain activity using EEG. S. reacts differently depending on how the colours correspond with his vision. In the case of a man without synesthesia, these reactions probably could not differ so dramatically. With the information on such schemes as opposed to psychological methods, we can clearly and reliably determine whether a person has the synesthetic ability. It should be considered to write a dedicated software, which on the basis of the above mentioned schemes could diagnose synesthesia.

\section{Acknowledgement}

Many thanks to S.

\section{References}

[1] Fisch, B. J., Fisch and Spehlmann's EEG Primer: Basic Principles of Digital and Analog EEG - Third revised and enlarged edition, New York: Elsevier (1999).

[2] Thompson, M., Thompson L., The Neurofeedback Book (2003).

[3] Tadeusiewicz, R., Using Neural Networks for Simplified Discovery of Some Psychological Phenomena. Artificial Intelligence and Soft Computing 6114, Lecture Notes in Artificial Intelligence, Springer-Verlag, Berlin - Heidelberg - New York (2010): 104.

[4] Tadeusiewicz, R., Computers in Psychology and Psychology in Computer Science, Proceedings of the 2010 International Conference on Computer Information Systems and Industrial Management Applications (CISIM) With Applications to Ambient Intelligence and Ubiquitous Systems. IEEE (2010): 34.

[5] Tadeusiewicz, R., Obciążenie psychiczne pracą - nowe wyzwania dla ergonomii pod redakcją Tadeusza Juliszewskiego, Halszki Ogińskiej i Macieja Złowodzki. Symulacyjne modele mózgu jako droga do poznania tajników umysłu, Komitet Ergonomii PAN (2011): 23.

[6] Tadeusiewicz, R., Informatyka i psychologia w społeczeństwie informacyjnym. Sieci neuronowe i inne systemy neurocybernetyczne jako narzędzia informatyczne o ciekawych zastosowaniach na gruncie psychologii, Wydawnictwa AGH Kraków (2011): 49.

[7] Mikołajewska, E., Mikołajewski, D., The prospects of brain - computer interface applications in children, Central European Journal of Medicine 9(1) (2014): 74.

[8] Mikołajewska, E., Mikołajewski, D., Integrated IT environment for people with disabilities: a new concept, Central European Journal of Medicine 9(1) (2014): 177.

[9] Hubbard, E M., Synaesthesia - A Window Into Perception, Thought and Language 12 (2001): 3.

[10] Beauchamp, M. S., \& Ro, T., Neural substrates of sound-touch synesthesia after a thalamic lesion, The Journal of neuroscience : the official journal of the Society for Neuroscience 28(50) (2008): 13696. DOI:10.1523/JNEUROSCI.3872-08.2008 
[11] Gertner, L., Henik, A., Reznik, D., \& Cohen Kadosh, R., Implications of number-space synesthesia on the automaticity of numerical processing, Cortex; a journal devoted to the study of the nervous system and behavior (2012): 1. DOI:10.1016/j.cortex.2012.03.019

[12] Muggleton, N., Tsakanikos, E., Walsh, V., \& Ward, J., Disruption of synaesthesia following TMS of the right posterior parietal cortex, Neuropsychologia 45(7) (2007): 1582. DOI:10.1016/j.neuropsychologia.2006.11.021

[13] Sinke, C., Neufeld, J., Emrich, H. M., Dillo, W., Bleich, S., Zedler, M., \& Szycik, G. R., Inside a synesthete's head: A functional connectivity analysis with grapheme-color synesthetes, 25. Neuropsychologia 50(14) (2012): 3363. DOI:10.1016/j.neuropsychologia.2012.09.015

[14] Brang, D., \& Ramachandran, V. S., Survival of the synesthesia gene: why do people hear colors and taste words? PLoS biology 9(11) (2011): e1001205. DOI:10.1371/journal.pbio.1001205

[15] Duch, W., Computational physics of the mind. Computer Physics Communications 97(12) (1996): 136. DOI:10.1016/0010-4655(96)00027-6

[16] Jewanski, J., Day, S.A., Ward, J., A colorful albino: the first documented case of synaesthesia, by Georg Tobias Ludwig Sachs in 1812, Journal of Cognitive Neuroscience 18( 3) (2009): 293.

[17] Mylopoulos, M. I., Ro, T., Synesthesia: a colorful word with a touching sound? Frontiers in psychology (2013). DOI: 10.3389/fpsyg.2013.00763

[18] Eagleman, D. M., Kagan, A. D., Nelson, S. S., Sagaram, D., Sarma, A. K., A standardized test battery for the study of synesthesia, Journal of Neuroscience Methods 159 (2007): 139.

[19] Brang, D., Hubbard, E. M., Coulson, S., Huang, M., Ramachandran, V.S., Magnetoencephalography reveals early activation of V4 in grapheme-color synesthesia. NeuroImage 53 (2010): 268 (Contents).

[20] Neufeld, J., Sinke, C., Zedler, M., Dillo, W., Emrich, H. M., Bleich, S., Szycik, G. R., Disinhibited feedback as a cause of synesthesia: evidence from a functional connectivity study on auditory-visual synesthetes, Neuropsychologia 50(7) (2012): 1471.

[21] Zamm, A., Schlaug, G., Eagleman, D. M., Loui, P., Pathways to seeing music: enhanced structural connectivity in colored-music synesthesia, NeuroImage 74 (2013): 359.

[22] Whitaker, K.,Kang, X., Herron, T., Woods, D., Robertson, L., Alvarez, B. D., White matter microstructure throughout the brain correlates with visual imagery in graphemecolor synesthesia, Neuroimage (2014). DOI: 10.1016/j.neuroimage.2013.12.054

[23] Wójcik, G. M., Mikołajewska, E., Mikołajewski, D., Wierzgała, P., Gajos, A., Smolira, M., Usefulness of EGI EEG system in brain computer interface research, Bio-Algorithms and Med-Systems 06/2013 9(2) (2013): 72.

[24] EGI; GeoSource 2.0 Technical Manual (2011).

[25] Pascual-Marqui, R. D., Standardized low resolution brain electromagnetic tomography (sLORETA): technical details. Methods \& Findings in Experimental \& Clinical Pharmacology 24D (2002): 5 .

[26] EGI; NetStation Waveforms Technical Manual (2011). 